\title{
Dynamics of non-expansive maps on strictly convex Banach spaces
}

\author{
Bas Lemmens* \\ Mathematics Institute, University of Warwick \\ CV4 7AL Coventry, United Kingdom \\ E-mail: lemmens@maths.warwick.ac.uk \\ Onno van Gaans ${ }^{\dagger}$ \\ Mathematical Institute, Leiden University \\ P.O. Box 9512, 2300 RA Leiden,The Netherlands \\ E-mail: vangaans@math.leidenuniv.nl.
}

\begin{abstract}
This paper concerns the dynamics of non-expansive maps on strictly convex finite dimensional normed spaces. By using results of Edelstein and Lyubich, we show that if $X=\left(\mathbb{R}^{n},\|\cdot\|\right)$ is strictly convex and $X$ has no 1-complemented Euclidean plane, then every bounded orbit of a non-expansive map $f: X \rightarrow X$, converges to a periodic orbit. By putting extra assumptions on the derivatives of the norm, we also show that the period of each periodic point of a non-expansive map $f: X \rightarrow X$ is the order, or, twice the order of a permutation on $n$ letters. This last result generalizes a theorem of Sine, who proved it for $\ell_{p}^{n}$ where $1<p<\infty$ and $p \neq 2$. To obtain the results we analyze the ranges of non-expansive projections, the geometry of 1-complemented subspaces, and linear isometries on 1-complemented subspaces.
\end{abstract}

\section{Introduction}

A frequently addressed problem in non-linear functional analysis is to describe the asymptotic behavior of the iterates of non-expansive maps $f: X \rightarrow$

${ }^{*}$ B. Lemmens acknowledges the support by Marie Curie Intra European Fellowship (MEIF-CT-2005-515391) of the European Commission.

†O van Gaans acknowlegdes the support by "Vidi subsidie" (639.032.510) of the Netherlands Organisation for Scientific Research (N.W.O.). 
$X$ on a real Banach space, see for instance $[1,3,6,8,22,24,25]$. This problem is already highly non-trivial in finite dimensional normed spaces. It is known $[14,21,27]$, for example, that if $\mathbb{R}^{n}$ is equipped with a polyhedral norm that has $N$ facets, then every bounded orbit of a non-expansive map $f: \mathbb{R}^{n} \rightarrow \mathbb{R}^{n}$ converges to a periodic orbit of $f$ whose period does not exceed $\max _{k} 2^{k}\left(\begin{array}{c}m \\ k\end{array}\right)$, where $m=N / 2$. Important examples of polyhedral norms on $\mathbb{R}^{n}$ are the $\ell_{\infty}$-norm, $\|x\|_{\infty}=\max _{i}\left|x_{i}\right|$, and the $\ell_{1}$-norm, $\|x\|_{1}=\sum_{i}\left|x_{i}\right|$. For the $\ell_{\infty}$-norm Nussbaum [21] has conjectured that the optimal upper bound for the possible periods of periodic points of non-expansive maps $f: \mathbb{R}^{n} \rightarrow \mathbb{R}^{n}$ is $2^{n}$. At present, however, this conjecture is proved for $n=1$, 2 and 3 only, see $[15,17]$. For the $\ell_{1}$-norm a complete characterization of the set of possible periods of periodic points of non-expansive maps in terms of arithmetical and combinatorial constraints was obtained in [13] (see also [23]).

Knowing these results the following problem is particularly interesting: Identify those norms on $\mathbb{R}^{n}$ that have the property that for every nonexpansive map $f: \mathbb{R}^{n} \rightarrow \mathbb{R}^{n}$ each bounded orbit converges to a periodic orbit. Obviously the $\ell_{2}$-norm does not possess this property as every rotation in the plane about an irrational angle is an isometry. On the other hand, Sine [25] has proved that the $\ell_{p}$-norms on $\mathbb{R}^{n}$ have this property if $1<p<\infty$ and $p \neq 2$. Moreover, he showed, in that case, that the period of each periodic point is an element of the set

$$
\begin{aligned}
\mathcal{S}(n)=\{p \in \mathbb{N}: p & =\operatorname{lcm}\left(p_{1}, \ldots, p_{k}\right) \text { or } p=2 \operatorname{lcm}\left(p_{1}, \ldots, p_{k}\right) \\
& \text { for some integers } \left.1 \leq p_{1}, \ldots, p_{k} \leq n \text { with } \sum_{i=1}^{k} p_{i} \leq n\right\} .
\end{aligned}
$$

In other words, each period is the order, or twice the order, of an element of the symmetric group on $n$ letters.

Our aim in this paper is to generalize Sine's result to more general strictly convex norms on $\mathbb{R}^{n}$. Among others, we show that if $\left(\mathbb{R}^{n},\|\cdot\|\right)$ is a strictly convex normed space that does not contain a 1-complemented Euclidean plane, then every bounded orbit of a non-expansive map converges to a periodic orbit. We note that this result is sharp. Indeed, if $\left(\mathbb{R}^{n},\|\cdot\|\right)$ is a strictly convex space with a 1-complemented Euclidean plane $E$, then one can compose the norm one projection onto $E$ with an irrational rotation on $E$ to obtain a non-expansive map for which most bounded orbits do not converge to periodic orbits. Under additional assumptions on the derivatives of the norm we also show that the possible periods of the periodic points are elements of $\mathcal{S}(n)$. Examples, other than the classical $\ell_{p}$-spaces with 
$1<p<\infty$ and $p \neq 2$, include vector valued $\ell_{p}$-spaces such as $\ell_{p}^{n}\left(\ell_{q}^{m}\right)$ for $p, q \geq 2$ and $1<p, q<2$, and certain Orlicz spaces. To obtain the results we shall initially study non-expansive maps on possibly infinite dimensional Banach spaces and subsequently specialize to the finite dimensional case. We emphasize that the results presented here depend on the assumption that the non-expansive map is defined on the whole Banach space, and that, in general, non-expansive maps defined on a proper subset of a Banach space need not admit a non-expansive extension to the whole space.

\section{Non-expansive projections}

In the sequel $X$ will denote a real Banach space. A map $f: A \rightarrow X$, with $A \subseteq X$, is called non-expansive if $\|f(x)-f(y)\| \leq\|x-y\|$ for all $x, y \in A$. It is said to be an isometry if equality holds for all $x, y \in A$. A map $\tau: X \rightarrow X$ is called a projection if $\tau^{2}(x)=\tau(x)$ for all $x \in X$. (We note that a projection need not be linear in general and is often called a retraction in the point-set topology literature.) To analyze the asymptotic behavior of the orbits, $\mathcal{O}(x)=\left\{f^{k}(x): k=0,1,2, \ldots\right\}$, one needs to understand the structure of the $\omega$-limit sets,

$$
\omega(x)=\left\{y \in X: f^{k_{i}}(x) \rightarrow y \text { for some }\left\{k_{i}\right\} \text { with } k_{i} \rightarrow \infty\right\} .
$$

We define the attractor of $f: X \rightarrow X$ by

$$
\Omega_{f}=\bigcup_{x \in X} \omega(x) .
$$

If $\mathcal{O}(x)$ is pre-compact and $f: X \rightarrow X$ is continuous, then $\omega(x)$ is non-empty and $f(\omega(x))=\omega(x)$. If $f$ is also non-expansive then the restriction of $f$ to $\omega(x)$ is an isometry (cf. [8]). In a separable Banach space, $\Omega_{f}$ is the range of a non-expansive projection, if $f: X \rightarrow X$ is non-expansive and every orbit of $f$ is pre-compact, as the following proposition shows.

Proposition 2.1. If $X$ is a separable Banach space and $f: X \rightarrow X$ is a non-expansive map such that $\mathcal{O}(x)$ is pre-compact for each $x \in X$, then there exists a non-expansive projection $\tau: X \rightarrow X$ onto $\Omega_{f}$ and the restriction of $f$ to $\Omega_{f}$ is an isometry.

The proof of this proposition uses the following lemma, which is proved in a similar way as the classical Arzela-Ascoli theorem. 
Lemma 2.2. If $X$ is a separable Banach space and $f: X \rightarrow X$ is a nonexpansive map such that $\mathcal{O}(x)$ is pre-compact for each $x \in X$, then every subsequence of $\left\{f^{k}\right\}$ has a convergent subsequence that converges uniformly on compact subsets of $X$.

Proof. As $X$ is a separable Banach space, there exists a countable dense subset $Y=\left\{y_{k}: k \in \mathbb{N}\right\}$ in $X$. Remark that $\left\{f^{k}\left(y_{1}\right)\right\}$ has a convergent subsequence, say $\left\{f^{k_{1, i}}\left(y_{1}\right)\right\}$, since $\mathcal{O}\left(y_{1}\right)$ has a compact closure. Similarly $\left\{f^{k_{1, i}}\left(y_{2}\right)\right\}$ has a convergent subsequence, $\left\{f^{k_{2, i}}\left(y_{2}\right)\right\}$. By repeating the argument indefinitely, we obtain for each $m \in \mathbb{N}$ a subsequence $\left\{f^{k_{m, i}}\right\}$ such that $\left\{f^{k_{m, i}}\left(y_{l}\right)\right\}$ converges for each $l \leq m$. Now we remark that the diagonal sequence $\left\{f^{k_{i, i}}\right\}$ has the property that $\left\{f^{k_{i, i}}\left(y_{m}\right)\right\}$ converges for each $m \in \mathbb{N}$.

For $x \in X$ and $\varepsilon>0$ there exists $y \in Y$ such that $\|x-y\|<\varepsilon$. As $f$ is non-expansive, we deduce that $\left\|f^{k_{i, i}}(x)-f^{k_{j, j}}(x)\right\| \leq \| f^{k_{i, i}}(x)-$ $f^{k_{i, i}}(y)\|+\| f^{k_{i, i}}(y)-f^{k_{j, j}}(y)\|+\| f^{k_{j, j}}(x)-f^{k_{j, j}}(y)\|\leq 2 \varepsilon+\| f^{k_{i, i}}(y)-f^{k_{j, j}}(y) \|$. Obviously the second term at the right hand side converges to 0 as $i, j \rightarrow \infty$, so that $\left\{f^{k_{i, i}}(x)\right\}$ is a Cauchy sequence in $X$ and hence it converges.

To show that the convergence is uniform on compact subsets of $X$, we let $C \subseteq X$ be compact and $\left\{z_{1}, \ldots, z_{r}\right\} \subseteq Y$ be such that $C \subseteq \cup_{i} B\left(z_{i}, \varepsilon\right)$, where $B\left(z_{i}, \varepsilon\right)$ is the open ball with radius $\varepsilon$ around $z_{i}$. By the same argument as in the previous paragraph we know for each $x \in C$ that there exists $1 \leq s \leq r$ such that

$$
\left\|f^{k_{i, i}}(x)-f^{k_{j, j}}(x)\right\| \leq 2 \varepsilon+\left\|f^{k_{i, i}}\left(z_{s}\right)-f^{k_{j, j}}\left(z_{s}\right)\right\| .
$$

Now let $I \in \mathbb{N}$ be such that

$$
\max _{1 \leq s \leq r}\left\|f^{k_{i, i}}\left(z_{s}\right)-f^{k_{j, j}}\left(z_{s}\right)\right\|<\varepsilon
$$

for all $i, j \geq I$. This implies that $\left\|f^{k_{i, i}}(x)-f^{k_{j, j}}(x)\right\| \leq 3 \varepsilon$ for all $i, j \geq I$ and $x \in C$, and hence $\left\{f^{k_{i, i}}\right\}$ converges uniformly on $C$.

Let us now prove Proposition 2.1.

Proof of Proposition 2.1. By Lemma 2.2 there exists a subsequence $\left\{f^{k_{i}}\right\}$ of $\left\{f^{k}\right\}$ that converges uniformly on compact subsets of $X$. By passing to a subsequence and applying the lemma again we may assume that $k_{i_{j}+1}-k_{i_{j}} \rightarrow$ $\infty$, as $j \rightarrow \infty$, and $\left\{f^{k_{i_{j}+1}-k_{i_{j}}}\right\}$ converges uniformly on compact subsets of $X$. Define $\tau: X \rightarrow X$ by

$$
\tau(x)=\lim _{j \rightarrow \infty} f^{k_{i_{j}+1}-k_{i_{j}}}(x) \quad \text { for each } x \in X .
$$


To see that $\tau$ is a projection onto $\Omega_{f}$ we first remark that $\tau(x) \in \Omega_{f}$ for each $x \in X$, so that $\tau(X) \subseteq \Omega_{f}$. Now let $y \in \Omega_{f}$. It follows from [8, Theorem 1] that $y \in \omega(y)$ and, as $\mathcal{O}(y)$ has a compact closure, $f(\omega(y))=\omega(y)$. Therefore there exists for each $k \in \mathbb{N}$ a point $y_{k} \in \omega(y)$ such that $f^{k}\left(y_{k}\right)=y$. Denote by $g$ the point-wise limit of $\left\{f^{k_{i}}\right\}$ and let $\left\{y_{l_{j}}\right\}$ be a convergent subsequence of $\left\{y_{k_{i}}\right\}$ with limit say $z \in \omega(y)$. (Such a subsequence exists as $\omega(y)$ is compact.) Clearly

$$
\left\|f^{l_{j}}\left(y_{l_{j}}\right)-g(z)\right\| \leq\left\|f^{l_{j}}\left(y_{l_{j}}\right)-g\left(y_{l_{j}}\right)\right\|+\left\|g\left(y_{l_{j}}\right)-g(z)\right\| \rightarrow 0,
$$

as $j \rightarrow \infty$, since $\left\{f^{l_{j}}\right\}$ converges uniformly to $g$ on $\omega(y)$. Thus, we find that $g(z)=y$.

Remark that

$$
f^{k_{i_{j}+1}}(z)=f^{k_{i_{j}+1}-k_{i_{j}}}\left(f^{k_{i_{j}}}(z)\right)
$$

for each $i \in \mathbb{N}$. The left hand side converges to $g(z)$, whereas the right hand side converges to $\tau(g(z))$. Therefore $\tau(y)=y$ for all $y \in \Omega_{f}$ and hence $\tau$ is a non-expansive projection onto $\Omega_{f}$.

It is now easy to deduce that $f$ is an isometry on $\Omega_{f}$, as $\|x-y\|=\| \tau(x)-$ $\tau(y)\left\|\leq \lim _{j \rightarrow \infty}\right\| f^{k_{i_{j}+1}-k_{i_{j}}}(x)-f^{k_{i_{j}+1}-k_{i_{j}}}(y)\|\leq\| f(x)-f(y)\|\leq\| x-y \|$ for all $x, y \in \Omega_{f}$.

A Banach space $X$ is said to be strictly convex if the unit sphere, $S_{X}=$ $\{x \in X:\|x\|=1\}$, does not contain a straight-line segment, or, equivalently, $\|x+y\|=\|x\|+\|y\|$ and $y \neq 0$ implies $x=(\|x\| /\|y\|) y$. It has been proved by Edelstein [11, Proposition 2] that if $f: X \rightarrow X$ is a non-expansive map on a strictly convex Banach space and $f$ is an isometry on $A \subseteq X$, then $f$ preserves convex combinations on the convex hull of $A$, i.e., $f(\lambda x+(1-$ $\lambda) y)=\lambda f(x)+(1-\lambda) f(y)$ for all $x, y \in A$ and $0 \leq \lambda \leq 1$. A combination of Edelstein's result with Proposition 2.1 immediately yields the following corollary.

Corollary 2.3. If $X$ is a strictly convex separable Banach space and $f: X \rightarrow$ $X$ is a non-expansive map such that $\mathcal{O}(x)$ is pre-compact for each $x \in X$, then $\Omega_{f}$ is convex.

Proof. Let $\tau: X \rightarrow X$ be the non-expansive projection onto $\Omega_{f}$ from Proposition 2.1. For each $x, y \in \Omega_{f}$ and $0 \leq \lambda \leq 1$ we have that $\tau(\lambda x+(1-\lambda) y)=$ $\lambda \tau(x)+(1-\lambda) \tau(y)=\lambda x+(1-\lambda) y$, so that $\lambda x+(1-\lambda) y \in \Omega_{f}$.

Under the assumptions in Corollary 2.3 we also have that $f: X \rightarrow X$ has a fixed point in $\Omega_{f}$. Indeed, $f(\omega(x))=\omega(x)$ and $\omega(x)$ is non-empty, as $f$ 
is continuous and $\mathcal{O}(x)$ has a compact closure. Thus, by Edelstein's result, $f(\overline{\operatorname{conv}}(\omega(x))) \subseteq \overline{\operatorname{conv}}(f(\omega(x)))=\overline{\operatorname{conv}}(\omega(x))$. But $\overline{\operatorname{conv}}(\omega(x))$ is compact, as $\omega(x)$ is compact (see [10, Theorem V.2.6]) and therefore $f: X \rightarrow X$ has a fixed point by the Schauder fixed point theorem. This result, however, also holds in finite dimensional normed spaces, and, more generally, under mild compactness conditions on $f$. It is not hard to show the following assertion.

Lemma 2.4. Let $X$ be a strictly convex separable Banach space. If $f: X \rightarrow$ $X$ is a non-expansive map such that $\mathcal{O}(x)$ is pre-compact for each $x \in X$ and $f(0)=0$, then there exists a unique linear extension of the restriction of $f$ to $\Omega_{f}$ to the closure of $\operatorname{span}\left(\Omega_{f}\right)$ and this extension is a surjective isometry.

Proof. As $0 \in \Omega_{f}$, it follows from Edelstein [11, Proposition 2] that the restriction of $f$ to $\Omega_{f}$ has a unique linear extension $F$ to the closure of $\operatorname{span}\left(\Omega_{f}\right)$. To see that $F$ is an isometry, we use the equality $\operatorname{span}\left(\Omega_{f}\right)=$ $\left\{\alpha x-\beta y: \alpha, \beta \geq 0\right.$ and $\left.x, y \in \Omega_{f}\right\}$, which is not hard to show. Let $z=$ $\alpha x-\beta y \in \Omega_{f}$, with $\alpha, \beta \geq 0$ and $x, y \in \Omega_{f}$. Take $M>\alpha, \beta$ and put $a=\alpha x / M$ and $b=\beta y / M$. Since $\Omega_{f}$ is convex and $0 \in \Omega_{f}$, we know that $a, b \in \Omega_{f}$, so that $\|F(z)\|=M\|f(a)-f(b)\|=M\|a-b\|=\|z\|$, due to Proposition 2.1. Finally, $F$ is surjective, as $f$ maps $\Omega_{f}$ onto $\Omega_{f}$ and $F$ is linear.

A subspace $Y \subseteq X$ is called 1-complemented in $X$ if it is the range of a linear non-expansive (norm one) projection. Also recall that a Banach space $X$ is smooth if for each $(x, y) \in S_{X} \times S_{X}$,

$$
\lim _{t \rightarrow 0} \frac{\|x+t y\|-\|x\|}{t}
$$

exists. If the limit in (2) is uniform for $(x, y) \in S_{X} \times S_{X}$, then $X$ is said to be uniformly smooth. Now suppose that $f: X \rightarrow X$ is a non-expansive map on a strictly convex separable Banach space and that $X$ is uniformly smooth. If each orbit of $f$ is pre-compact, $\Omega_{f}$ has a non-empty relative interior as a subset of $\operatorname{span}\left(\Omega_{f}\right)$ and $0 \in \Omega_{f}$, then we shall show that $\operatorname{span}\left(\Omega_{f}\right)$ is 1complemented in $X$. To prove this we use the following observation by Sine [25]. As Sine omits most of the details of the proof, we provide them here.

Proposition 2.5. Let $X$ be a uniformly smooth Banach space and $A$ be a convex subset of $X$ with non-empty relative interior as a subset of $\operatorname{span}(A)$ and $0 \in A$. If there exists a non-expansive projection $\tau: X \rightarrow X$ onto $A$, then $\operatorname{span}(A)$ is 1-complemented in $X$. 
Proof. Before we start the proof we like to point out that under the assumptions $\operatorname{span}(A)$ must be closed, as the range of a non-expansive projection is closed. To prove the proposition we need the notion of a sunny projection. A projection $\tau: X \rightarrow X$ is called sunny if for each $x, y \in X$ with $\tau(x)=y$ we have that $\tau(y+\lambda(x-y))=y$ for all $\lambda \geq 0$. It has been shown by Bruck [7, Theorem 2] that if $X$ is a uniformly smooth Banach space and $A \subseteq X$ is the range of a non-expansive projection, then there exists a sunny non-expansive projection $s: X \rightarrow X$ onto $A$.

For $x \in A$ and $y \in \operatorname{span}(A)$ with $x \neq y$, let $x+H_{x}(y)$ denote the hyperplane through $x$ tangent to the ball $B(y,\|x-y\|)$ centered at $y$ with radius $\|x-y\|$. In other words $H_{x}(y)$ is the null space of $J_{X}(x-y)$, where $J_{X}: X \rightarrow X^{*}$ is the duality map. Recall that as $X$ is uniformly smooth, $X^{*}$ is uniformly convex, so that $J_{X}$ is a single valued continuous map. In fact, $J_{X}(z)$ is the unique functional such that $\left\|J_{X}(z)\right\|=\|z\|$ and $J_{X}(z)(z)=$ $\|z\| \cdot\left\|J_{X}(z)\right\|$.

For each $x \in A$ write

$$
N_{x}=\cap\left\{H_{x}(y): y \in \operatorname{span}(A) \text { and } y \neq x\right\} .
$$

We note that if $x \in A$ and $y \in \operatorname{span}(A)$ with $x \neq y$, then $H_{x}(y)=H_{x}(z)$ for all $z \in\{x+\lambda(y-x): \lambda>0\}$, as $J_{X}\left(x-(x+\lambda(y-x))=\lambda J_{X}(x-y)\right.$ for all $\lambda>0$. Since $A$ is convex, this gives for each $x \in \operatorname{relint}(A)$ (relative interior of $A$ ) that

$$
\begin{aligned}
N_{x} & =\cap\left\{H_{x}(y): y \in \operatorname{relint}(A) \text { and } y \neq x\right\} \\
& =\cap\left\{H_{x}(y): y \in \operatorname{relint}(A), y \neq x \text { and }\|y-x\|<\alpha\right\}
\end{aligned}
$$

for any $\alpha>0$. Moreover, we have that $N_{x}=N_{y}$ for all $x, y \in \operatorname{relint}(A)$, since $\operatorname{span}(A)$ is a subspace containing $A$. We write $N=N_{x}$ for $x \in \operatorname{relint}(A)$ Furthermore we define $F(x)$ to be the affine subspace generated by $s^{-1}(\{x\})$ for $x \in \operatorname{relint}(A)$. We remark that $N \cap \operatorname{span}(A)=\{0\}$. Indeed, if $x \in$ $\operatorname{relint}(A)$ and there exists $y \in(x+N) \cap \operatorname{span}(A)$ with $y \neq x$, then $y-x \in$ $N \subseteq N_{x}(y)$, so that $y$ is in the hyperplane tangent to $B(y,\|y-x\|)$, which is impossible. The remainder of the proof is split up into two claims.

Claim 1. For each $x \in \operatorname{relint}(A), F(x)=x+N$. To show this claim we first prove that $F(x) \subseteq x+N$. Let $z \in s^{-1}(\{x\})$. As $s$ is a sunny non-expansive projection onto $A$, the half-line $\ell_{x, z}^{+}=\{x+\lambda(z-x): \lambda \geq 0\}$ does not intersect the open ball $B(y,\|y-x\|)$ for all $y \in A$. Let $\alpha>0$ so small that if $y \in A$ and $\|x-y\|<\alpha$, then the reflection of $y$ in $x$, given by $y^{\prime}=2 x-y$, is also in $A$. Next we show that $\ell_{x, z}^{-}=\{x+\lambda(z-x): \lambda \leq 0\}$ does not intersect the open ball $B(y,\|y-x\|)$ for all $y \in A$ with $\|x-y\|<\alpha$. Suppose, for the 
sake of contradiction, that there exists $y \in A$ such that $\|x-y\|<\alpha$ and $\ell_{x, z}^{-}$ intersects $B(y,\|y-x\|)$. Let $y^{\prime}$ be the reflection of $y$ in $x$. It is easy to see that the reflection of $B(y,\|x-y\|)$ in $x$ is equal to $B\left(y^{\prime},\left\|x-y^{\prime}\right\|\right)$. Indeed, if $z^{\prime}$ is the reflection of $z \in B(y,\|x-y\|)$ in $x$, then

$$
\left\|z^{\prime}-y^{\prime}\right\|=\|(2 x-z)-(2 x-y)\|=\|z-y\|<\|x-y\|=\left\|x-y^{\prime}\right\|,
$$

which proves one inclusion. The other inclusion follows by symmetry. This implies that $\ell_{x, z}^{+} \cap B\left(y^{\prime},\left\|x-y^{\prime}\right\|\right)$ is non-empty, which is a contradiction, as $y^{\prime} \in A$ and $s$ is a sunny non-expansive projection. Thus, we find that $l_{x, z}=\{x+\lambda(z-x): \lambda \in \mathbb{R}\}$ does not intersect $B(y,\|x-y\|)$ for all $y \in A$ with $\|x-y\|<\alpha$. As $N$ is a linear subspace, it thus follows from (3) that $F(x) \subseteq x+N$.

To prove the opposite inclusion, $x+N \subseteq F(x)$, let $z \in x+N$ and $z \neq x$. We note that $z-x \notin \operatorname{span}(A)$, as $N \cap \operatorname{span}(A)=\{0\}$. To show that $s(z)=x$, it suffices to show that $s(x+\varepsilon(z-x))=x$ for some $\varepsilon>0$, since $s$ is sunny. We may therefore assume that $s(z) \in \operatorname{relint}(A)$, as $x \in \operatorname{relint}(A)$. Suppose that $s(z) \neq x$. Then the 2-dimensional affine subspace $W$ through $z, x$ and $s(z)$ intersects $x+N$ in the line $l_{x, z}$, because $x, z \in x+N$ and $s(z) \notin x+N$. (Otherwise $s(z)-x \in N \cap \operatorname{span}(A)$.) The line $l_{s(z), z}$ is contained in $F(s(z)) \subseteq s(z)+N$, because $s(z) \in \operatorname{relint}(A)$. By translation, $l_{x, z-(s(z)-x)} \subseteq x+N$, so that $l_{x, z-(s(z)-x)}$ is contained in the intersection of $x+N$ and the affine plane $W$. Therefore $x, z$ and $z-(s(z)-x)$ are all on a line, which contradicts the fact that $s(z) \notin x+N$. Thus, we find that $s(z)=x$ and hence $F(x)=x+N$.

Claim 2. $\operatorname{span}(A) \oplus N=X$. There exists $x \in \operatorname{relint}(A)$ and a closed ball $B$ around $x$ such that $s(B) \subseteq \operatorname{relint}(A)$. For $y \in X$ with $s(y) \in \operatorname{relint}(A)$ we have that $y \in F(s(y))=s(y)+N$ by Claim 1, so that $y-s(y) \in N$. Hence $y=s(y)+(y-s(y)) \in \operatorname{span}(A) \oplus N$. Let $v \in X$ be arbitrary. Choose $\varepsilon>0$ so small that $x+\varepsilon v \in B$. Then $x+\varepsilon v=y_{1}+y_{2}$, where $y_{1} \in \operatorname{span}(A)$ and $y_{2} \in N$. This implies that $v=\left(\left(y_{1}-x\right)+y_{2}\right) / \varepsilon \in \operatorname{span}(A) \oplus N$.

By using Claim 2 we can define $P: X \rightarrow X$ to be the linear projection onto $\operatorname{span}(A)$ along $N$, so $P(u+v)=u$ for all $u \in \operatorname{span}(A)$ and $v \in N$. To complete the proof we show that $P$ is non-expansive. Let $x \in \operatorname{relint}(A)$ and $z \in X$ arbitrary. Pick $\varepsilon>0$ such that $(1-\varepsilon) x \in \operatorname{relint}(A)$ and $y=x+\varepsilon(P(z)-x) \in \operatorname{relint}(A)$. Then $x+\varepsilon(z-x)-y \in N \subset H_{y}((1-\varepsilon) x)$ and hence the line through $y$ and $x+\varepsilon(z-x)$ is tangent to the ball $B((1-$ $\varepsilon) x,\|(1-\varepsilon) x-y\|)$. In particular, $x+\varepsilon(z-x)$ is outside this ball, so that

$$
\|x+\varepsilon(z-x)-(1-\varepsilon) x\| \geq\|(1-\varepsilon) x-y\| .
$$

This implies that $\|\varepsilon z\| \geq\|\varepsilon P(z)\|$, and hence $P$ is non-expansive. 
If in Proposition 2.5 the relative interior of $A$ is empty, then $\operatorname{span}(A)$ need not be 1-complemented. Indeed, if $1 \leq p<\infty$ and $A=\left\{x \in \ell_{p}:\left|x_{i}\right| \leq\right.$ $2^{-i}$ for all $\left.i\right\}$, then $A$ is a closed convex set and the coordinatewise nearest point projection $\tau$ onto $A$ is non-expansive. That is, $\tau(x)_{i}=x_{i}$ if $\left|x_{i}\right| \leq 2^{-i}$ and $\tau(x)_{i}=\operatorname{sgn}\left(x_{i}\right) 2^{-i}$ if $\left|x_{i}\right|>2^{-i}$. The linear span of $A$ is a dense proper subspace of $\ell_{p}$ and therefore not 1-complemented. It appears to be unknown whether the closure of $\operatorname{span}(A)$ is always 1-complemented in a uniformly smooth Banach space $X$, if $A$ is a convex subset of $X$ containing 0 and $A$ is the range of a non-expansive projection.

By combining the results so far we obtain the following result.

Corollary 2.6. Let $X$ be a uniformly smooth strictly convex separable Banach space. If $f: X \rightarrow X$ is non-expansive such that $\mathcal{O}(x)$ is pre-compact for each $x \in X, f(0)=0$, and $\Omega_{f}$ has non-empty relative interior as a subset of $\operatorname{span}\left(\Omega_{f}\right)$, then $Y=\operatorname{span}\left(\Omega_{f}\right)$ is a 1-complemented subspace of $X$ and the restriction of $f$ to $\Omega_{f}$ extends to a linear isometry from $Y$ onto $Y$.

A 2-dimensional subspace $Y$ of a Banach space is called a Euclidean plane if $\left(Y,\|\cdot\|_{X}\right)$ is a Hilbert space. The following useful result is due to Lyubich [18, Theorem 4].

Theorem 2.7. If $X=\left(\mathbb{R}^{n},\|\cdot\|\right)$ has an infinite group of linear isometries, then $X$ contains a 1-complemented Euclidean plane.

By using Lyubich's theorem and the previous lemmas we now prove the following assertion.

Theorem 2.8. Let $X$ be a uniformly smooth strictly convex separable Banach space that contains no 1-complemented Euclidean plane and let $f: X \rightarrow$ $X$ be a non-expansive map with $f(0)=0$. If $\mathcal{O}(x)$ is pre-compact for each $x \in X$ and $\operatorname{dim} \operatorname{span}\left(\Omega_{f}\right)<\infty$, then there exists $p \in \mathbb{N}$ such that $|\omega(x)| \leq p$ for all $x \in X$.

Proof. As $X$ is a strictly convex separable Banach space, it follows from Corollary 2.3 that $\Omega_{f}$ is convex. Moreover, there exists a surjective linear isometry $F: \operatorname{span}\left(\Omega_{f}\right) \rightarrow \operatorname{span}\left(\Omega_{f}\right)$ that extends $f$ on $\Omega_{f}$ by Lemma 2.4. Since $\operatorname{span}\left(\Omega_{f}\right)$ is finite dimensional, the relative interior of $\Omega_{f}$ in this subspace is non-empty. Let $Y=\left(\operatorname{span}\left(\Omega_{f}\right),\|\cdot\|_{X}\right)$ and remark that as $X$ is uniformly smooth, $\operatorname{span}\left(\Omega_{f}\right)$ is 1-complemented in $X$ by Proposition 2.5. From Lyubich [18, Theorem 4] we deduce that $\Gamma=\{T: Y \rightarrow$ $Y \mid T$ linear isometry $\}$ is a finite group. Otherwise $Y$ would contain a 1complemented Euclidean plane $E$, and $E$ would also be 1-complemented in 
$X$, as $\operatorname{span}\left(\Omega_{f}\right)$ is 1-complemented, which contradicts the assumption in the theorem.

Let $p=|\Gamma|$ and $x \in X$. Suppose that $y \in \omega(x)$ and note that, as $f$ is continuous and $\mathcal{O}(x)$ is pre-compact, $f(\omega(x))=\omega(x)$. Moreover it follows from [8, Theorem 1] that $\omega(x)=\omega(y)$. Clearly $F^{k} \in \Gamma$ for each $k \geq 0$. Hence there exist $0 \leq k<m \leq p$ such that $F^{k}=F^{m}$. This implies that $F^{m-k}(y)=y$, so that $|\omega(x)|=|\omega(y)| \leq m-k \leq p$ and we are done.

It follows from Theorem 2.8 that, under the assumptions, every orbit converges to a periodic orbit, as each $\omega$-limit set is finite and itself a periodic orbit. To obtain estimates for the lengths of the periodic orbits, we need to better understand the 1-complemented subspaces of a Banach space and the surjective linear isometries acting on them.

\section{Isometries on 1-complemented subspaces}

For the classical $L_{p}$ spaces, with $1 \leq p<\infty$, it is well known that every 1-complemented subspace is isometrically isomorphic to an $L_{p}$ space (see $[2,5,9,26])$. Therefore if $1<p<\infty$ and $f: L_{p} \rightarrow L_{p}$, with $f(0)=0$ is a nonexpansive map such that each orbit of $f$ is pre-compact and $\operatorname{dim}\left(\operatorname{span} \Omega_{f}\right)=$ $n$, then $\left(\operatorname{span}\left(\Omega_{f}\right),\|\cdot\|_{p}\right)$ is isometrically isomorphic to $\ell_{p}^{n}$. In that case one can use Banach's [4] characterization of the linear isometries on $\ell_{p}$ for $1<p<\infty$ and $p \neq 2$, to conclude that the cardinality of each $\omega$-limit is the order, or twice the order, of an element of the symmetric group on $n$ letters. These facts were used by Sine in [25]. In general, however, a 1-complemented subspace in a Banach space need not be isometrically isomorphic to (a lower dimensional version of ) the original space. Nevertheless there are still ways to analyze the linear isometries acting on them.

To do this, we need to recall some additional notions from the theory of Banach spaces. Most of these notions can be found in [19]. Let $X$ be a Banach space with a (Schauder) basis $\left\{e_{i}\right\}$, so for each $x \in X$ there exists a unique sequence of scalars $\left\{\alpha_{i}\right\}$ such that $x=\sum_{i} \alpha_{i} e_{i}$. The basis is said to be 1-unconditional if for each $x=\sum_{i} \alpha_{i} e_{i} \in X$ we have that $\|x\|=\sup _{\vartheta}\left\|\sum_{i} \vartheta_{i} \alpha_{i} e_{i}\right\|$, where $\vartheta_{i} \in\{-1,1\}$ for each $i$. We denote by $\sigma(x)=\left\{i: \alpha_{i} \neq 0\right\}$ the support of $x$. If $\sigma(x)$ is finite, then we call $x$ simple. A subspace $Y \subseteq X$ is said to have a block basis if there exists a basis $\left\{y_{i}\right\}$ for $Y$ whose elements are disjointly supported, so $\sigma\left(y_{i}\right)$ and $\sigma\left(y_{j}\right)$ are disjoint for all $i \neq j$. The following notion was introduced in [16] and is particularly useful in the study of 1-complemented subspaces and linear isometries (see also [12]). 
Definition 3.1. Suppose that $X$ is a Banach space with a basis and for each $x, y \in X$ the function $N=N_{x, y}: \mathbb{R} \rightarrow \mathbb{R}$ is given by

$$
N(\alpha)=\|x+\alpha y\| \text { for all } \alpha \in \mathbb{R} .
$$

We say that $X$ reflects disjointness if for each $x, y \in X$ with $x \notin \operatorname{span}(y)$ the following conditions hold:

(i) the function $N$ is continuously differentiable;

(ii) if $x$ and $y$ are not disjoint in $X$ and $N^{\prime}(0)=0$, then $N^{\prime \prime}(\alpha)$ does not converge to 0 as $\alpha \rightarrow 0$ along any subset of $[0,1]$ of full measure;

(iii) if $x$ and $y$ are disjoint in $X$ and $y$ is simple, then $N^{\prime}(0)=0$ and $N^{\prime \prime}(\alpha)$ does converge to 0 as $\alpha \rightarrow 0$ along a subset of $[0,1]$ of full measure.

Although this definition looks somewhat technical, it has a natural geometric interpretation in finite dimensions in terms of the directional curvature of the unit sphere. In fact, the following statement holds.

Lemma 3.2. If $X=\left(\mathbb{R}^{n},\|\cdot\|\right)$ has a $C^{2}$ smooth norm and the standard basis is 1-unconditional, then $X$ reflects disjointness is equivalent to saying that at each $x \in S_{X}$ the normal curvature in the direction of $y$ is 0 if, and only if, $x$ and $y$ are disjoint.

Proof. Let $S^{n-1}$ denote the Euclidean sphere in $\mathbb{R}^{n}$ and $S_{X}^{r}$ denote the sphere with radius $r>0$ in $X$. Then the Gauss map, $\mathcal{N}: S_{X}^{r} \rightarrow S^{n-1}$, is given by

$$
\mathcal{N}(x)=\frac{\nabla\|x\|}{|\nabla\|x\||},
$$

where $|z|$ denotes the Euclidean norm of $z$. Let $V_{x}$ be the tangent space at $x \in S_{X}^{r}$. For $x \in S_{X}^{r}$ the Weingarten map $\mathcal{L}_{x}: V_{x} \rightarrow V_{x}$ is given by $\mathcal{L}_{x}(v)=-\nabla_{v} \mathcal{N}(x)$. The normal curvature of $S_{X}^{r}$ at $X$ in the direction $v \in V_{x}$ is given by

$$
\kappa_{x}(v)=\left\langle\mathcal{L}_{x}(v), v\right\rangle .
$$

Note that as $\mathcal{L}_{\lambda x}(v)=\lambda \mathcal{L}_{x}(v)$ for all $\lambda>0$, we have that $\kappa_{x}(y)=0$ if, and only if, $\kappa_{\lambda x}(y)=0$ for all $\lambda>0$.

As $X$ is $C^{2}$ smooth, we have for each $x, y \in X$ with $x \notin \operatorname{span}(y)$ that $N=N_{x, y}$ is $C^{2}$ and $N^{\prime}(\alpha)=\langle\nabla\|x+\alpha y\|, y\rangle$ and $N^{\prime \prime}(\alpha)=\left\langle H_{X}(x+\alpha y) y, y\right\rangle$, where $H_{X}$ is the Hessian of $x \mapsto\|x\|$. Note that

$$
\mathcal{L}_{x}(v)=-\nabla_{v} \mathcal{N}(x)=\frac{\left\langle\nabla\|x\|, H_{X}(x) v\right\rangle}{|\nabla\|x\||^{3}} \nabla\|x\|-\frac{H_{X}(x) v}{|\nabla\|x\||} .
$$


Thus,

$$
\left\langle H_{X}(x) v, v\right\rangle=\frac{\left\langle\nabla\|x\|, H_{X}(x) v\right\rangle}{|\nabla\|x\||^{2}}\langle\nabla\|x\|, v\rangle-\mid \nabla\|x\| \| \kappa_{x}(v) .
$$

Assume that $X$ satisfies the curvature condition. Suppose that $x, y \in X$, with $x \notin \operatorname{span}(y)$, are not disjoint and $N^{\prime}(0)=0$. Remark that $N^{\prime}(0)=0$ is equivalent to $\langle\nabla\|x\|, y\rangle=0$. As $N^{\prime \prime}(0)=\left\langle H_{X}(x) y, y\right\rangle$ and $y \in V_{x}$, we deduce from (4) that

$$
N^{\prime \prime}(0)=-|\nabla\|x\|| \kappa_{x}(y) .
$$

Let $v^{1}, \ldots, v^{n-1}$ be an orthonormal basis for $V_{x}$ consisting of the eigenvectors of $\mathcal{L}_{x}: V_{x} \rightarrow V_{x}$. Note that as $H_{X}(x)$ is positive semi-definite and symmetric, $\mathcal{L}_{x}$ is negative semi-definite and symmetric. Relabel the eigenvectors such that $\lambda_{1}(x)=\lambda_{2}(x)=\ldots=\lambda_{k}(x)=0$ and $\lambda_{i}(x)<0$ for all $i>k$. We write

$$
y=\sum_{i=1}^{k} a_{i} v^{i}+\sum_{i=k+1}^{n-1} a_{i} v^{i} .
$$

There exists $j>k$ such that $a_{j} \neq 0$ in (5). Indeed, if $a_{j}=0$ for all $j>k$, then $\mathcal{L}_{x}(y)=0$, so that $\kappa_{x}(y)=0$. But this implies that $\kappa_{x / r}(y)=0$, so that $x$ and $y$ are disjoint, which is a contradiction. As $a_{j} \neq 0$ for some $j>k$, we get that

$$
\begin{aligned}
N^{\prime \prime}(0) & =-\mid \nabla\|x\| \| \kappa_{x}(y) \\
& =-|\nabla\|x\||\left\langle\sum_{i} a_{i} \mathcal{L}_{x}\left(v^{i}\right), \sum_{i} a_{i} v^{i}\right\rangle \\
& =-|\nabla\|x\|| \sum_{i>k} \lambda_{i}(x)\left\langle a_{i} v^{i}, a_{i} v^{i}\right\rangle>0 .
\end{aligned}
$$

Thus Property (ii) in Definition 3.1 holds.

As $X$ has a 1-unconditional basis, we know that $\sigma(x)=\sigma\left(J_{X}(x)\right)=$ $\sigma(\nabla\|x\|)$. Thus, if $x$ and $y$ are disjoint, then $\nabla\|x\|$ and $y$ are disjoint, so that $N^{\prime}(0)=\langle\nabla\|x\|, y\rangle=0$. To see that $N^{\prime \prime}(0)=0$, we remark that as $x$ and $y$ are disjoint, $\kappa_{x}(y)=\kappa_{x /\|x\|}(y)=0$, so that $N^{\prime \prime}(0)=-\mid \nabla\|x\| \| \kappa_{x}(y)=0$.

To prove the opposite implication we suppose that $X$ reflects disjointness. Assume that $x \in S_{X}, y \in V_{x}$, and $x$ and $y$ not disjoint. As $y \in V_{x}, N^{\prime}(0)=0$, so that $N^{\prime \prime}(0) \neq 0$. As $N^{\prime \prime}(0)=-|\nabla\|x\|| \kappa_{x}(y)$, we conclude that $\kappa_{x}(y) \neq 0$. On the other hand, if $x$ and $y$ are disjoint, then $N^{\prime \prime}(0)=0$ and hence $\kappa_{x}(y)=0$. 
The next result from [16] shows the relevance of Definition 3.1 in the context of 1-complemented subspaces.

Theorem 3.3. If $X$ is a $C^{2}$ smooth Banach space with a 1-unconditional basis, and $X$ reflects disjointness, then every 1-complemented subspace has a block basis.

Recall that a Banach space is $C^{2}$ smooth if the norm function is twice continuously Frechet differentiable. The proof of the Theorem 3.3 relies on the following observation [16, Lemma 3.5] concerning convex functions.

Lemma 3.4. Let $\varphi: \mathbb{R} \rightarrow[0, \infty)$ and $\psi: \mathbb{R} \rightarrow[0, \infty)$ be convex functions that are continuously differentiable and assume that $\varphi^{\prime}$ and $\psi^{\prime}$ are absolutely continuous on $[0,1]$. If $\varphi(0)=\psi(0)$ and $\varphi(\alpha) \leq \psi(\alpha)$ for all $\alpha \in[0, \infty)$, then

(i) $\varphi^{\prime}(0)=\psi^{\prime}(0)$,

(ii) the set consisting of those $\alpha \in(0, \infty)$ for which $\varphi^{\prime \prime}(\alpha)$ and $\psi^{\prime \prime}(\alpha)$ exist and $\varphi^{\prime \prime}(\alpha) \leq \psi^{\prime \prime}(\alpha)$ has positive measure on each interval $(0, \delta)$ for $\delta>0$,

(iii) for every $C>0$ the measure of the set consisting of those $\alpha \in[0,1]$ for which $\psi^{\prime \prime}(\alpha)$ exists and $\psi^{\prime \prime}(\alpha) \leq C$ is strictly smaller than 1 , whenever $\varphi^{\prime \prime}(\alpha) \rightarrow \infty$, as $\alpha \rightarrow 0$ along a subset of full measure.

We shall use Lemma 3.4 again to prove the following proposition.

Proposition 3.5. Let $X$ be a $C^{2}$ smooth Banach space with 1-unconditional basis and suppose that $X$ reflects disjointness. If $Y \subseteq X$ is a 1-complemented subspace of $X$ with a block basis $\left\{y_{i}\right\}$, then every linear isometry $T: Y \rightarrow Y$ is disjointness preserving with respect to $\left\{y_{i}\right\}$.

Proof. Let $P$ denote a non-expansive linear projection onto $Y$. First, consider $u \in Y$ and $v \in X$ with $u \neq 0, v$ simple, and $u$ and $v$ disjoint. Let $\varphi: \mathbb{R} \rightarrow[0, \infty)$ and $\psi: \mathbb{R} \rightarrow[0, \infty)$ be given by $\varphi(\alpha)=\|T P u+\alpha T P v\|$ and $\psi(\alpha)=\|u+\alpha v\|$ for all $\alpha \in \mathbb{R}$, respectively. Then $\varphi$ and $\psi$ are convex, $\varphi(\alpha) \leq \psi(\alpha)$ for all $\alpha \in \mathbb{R}$, and $\varphi(0)=\psi(0)$, as $T$ is an isometry. Moreover, $\varphi$ and $\psi$ are $C^{2}$ functions, as $X$ is a $C^{2}$ Banach space. Since $X$ reflects disjointness, we have $\psi^{\prime}(0)=0$ and $\psi^{\prime \prime}(\alpha) \rightarrow 0$ along a subset of $[0,1]$ of full measure. By Lemma 3.4 we get that $\varphi^{\prime}(0)=0$ and $\varphi^{\prime \prime}(0)=0$, as $\varphi^{\prime \prime}$ is continuous. Now the second property of Definition 3.1 implies that $T P u$ and $T P v$ are disjoint in $X$. But $T P u$ and $T P v$ are in $Y$, so that $T P u=\sum_{i} \alpha_{i} y_{i}$ 
and $T P v=\sum_{i} \beta_{i} y_{i}$, and hence $T P u$ and $T P v$ are also disjoint with respect to $\left\{y_{i}\right\}$.

Next, let $x, y \in Y$ be disjoint with respect to $\left\{y_{i}\right\}$. Choose a sequence $\left\{v^{n}\right\}_{n}$ in $X$ that converges to $y$ with each $\sigma\left(v^{n}\right) \subseteq \sigma(y)$ and each $v^{n}$ simple. As $\left\{y_{i}\right\}$ is a block basis, $x$ and $y$ are also disjoint in $X$ and hence $x$ and $v^{n}$ are disjoint for all $n$. By the first part of the proof we obtain that TPx and $T P v^{n}$ are disjoint with respect to $\left\{y_{i}\right\}$. As $T P v^{n}$ converges to $T P y$, it follows that $T x=T P x$ and $T y=T P y$ are disjoint with respect to $\left\{y_{i}\right\}$. Hence $T$ is disjointness preserving with respect to $\left\{y_{i}\right\}$.

Surjective disjointness preserving isometries have a special form.

Lemma 3.6. If $X$ is a Banach space with a normalized basis $\left\{e_{i}\right\}$ and $T: X \rightarrow X$ is a surjective disjointness preserving isometry, then there exists a permutation $\pi$ and a sequence $\left\{\vartheta_{i}\right\}$ in $\{-1,1\}$ such that

$$
T x=\sum_{i} \alpha_{i} \vartheta_{\pi(i)} e_{\pi(i))} \quad \text { for all } x=\sum_{i} \alpha_{i} e_{i} \in X .
$$

Proof. First we remark that surjective isometries on Banach spaces are linear by the Mazur-Ulam theorem [20]. Let $\left\{e_{i}\right\}$ be a normalized basis for $X$. We claim that for each $j$ there exists a unique $i$ and $\beta_{i} \in\{-1,1\}$ such that $T e_{j}=\beta_{i} e_{i}$. Indeed, if $v \in \operatorname{span}\left\{e_{i}: i \in \sigma\left(T e_{j}\right)\right\}$ and $0 \neq v \neq \beta T e_{j}$ for all $\beta \in \mathbb{R}$, then there exists $u=\sum_{i} \gamma_{i} e_{i} \in X$ with $T u=v$. Note that $T e_{k}$ and $T e_{m}$ are disjoint for all $k \neq m$. Therefore $T u=T\left(\sum_{i} \gamma_{i} e_{i}\right)=$ $\gamma_{j} T e_{j}$, as $\sigma(T u)=\sigma(v) \subseteq \sigma\left(T e_{j}\right)$. Thus, $v=T u=\gamma_{j} T e_{j}$, which is a contradiction. Hence $\left|\sigma\left(T e_{j}\right)\right|=1$ and $T e_{j}=\beta_{i} e_{i}$, where $\beta_{i} \in\{-1,1\}$, since $\left\|e_{j}\right\|=\left\|T e_{j}\right\|=\left|\beta_{i}\right|\left\|e_{i}\right\|$. Now let $\pi$ be the permutation that asigns to $j$ the unique element in $\sigma\left(T e_{j}\right)$. Clearly $T\left(\sum_{j} \alpha_{j} e_{j}\right)=\sum_{j} \alpha_{j} \vartheta_{\pi(j)} e_{\pi(j)}$ for all $\sum_{j} \alpha_{j} e_{j} \in X$.

A linear operator of the form (6) is called a signed permutation. If a Banach space $X$ with a 1-unconditional basis $\left\{x_{i}\right\}$ is uniformly smooth, then it is reflexive (see [19, Theorem 5.5.13]) and then the coordinate functionals $\left\{x_{i}^{*}\right\}$ are a 1-unconditional basis in $X^{*}$ (see [19, Corollary 4.4.16]). We can now prove our main result.

Theorem 3.7. Let $X$ be a uniformly smooth strictly convex Banach space with a 1-unconditional basis. Suppose that $f: X \rightarrow X$ is a non-expansive map such that $f(0)=0, \mathcal{O}(x)$ is pre-compact for every $x \in X$, and the relative interior of $\Omega_{f}$ as a subset of $\operatorname{span}\left(\Omega_{f}\right)$ is non-empty. If either $X$ or $X^{*}$ is $C^{2}$ smooth and reflects disjointness, then $Y=\operatorname{span}\left(\Omega_{f}\right)$ has a 
block basis, $\left.f\right|_{\Omega_{f}}$ extends to a linear isometry $T$ in $Y$, and $T$ is a signed permutation operator with respect to the block basis of $Y$. Moreover, if $\operatorname{dim} \operatorname{span}\left(\Omega_{f}\right)=n<\infty$, then $|\omega(x)| \in \mathcal{S}(n)$ for each $x \in X$.

Proof. Due to Corollary 2.6 the subspace $Y=\operatorname{span}\left(\Omega_{f}\right)$ is 1-complemented and the restriction of $f$ to $\Omega_{f}$ extends to a linear isometry $T$ from $Y$ onto $Y$. Let $P$ denote a nonexpansive projection onto $Y$. If $X$ is $C^{2}$ smooth and reflects disjointness, then it follows from Theorem 3.3 and Proposition 3.5 that $T: Y \rightarrow Y$ is disjointness preserving with respect to the block basis of $Y$. Therefore $T$ is a signed permutation operator by Lemma 3.6.

If $X^{*}$ is $C^{2}$-smooth and reflects disjointness, then we let $Y^{\prime}$ denote the range of the adjoint $P^{*}$ of $P$. Thus $Y^{\prime}$ is 1-complemented in $X^{*}$ and, according to Theorem 3.3, $Y^{\prime}$ has a block basis $\left\{y_{i}^{\prime}\right\}$. We now show that $Y$ has a block basis. Since $X$ is reflexive and strictly convex, there exists for each non-zero $x^{*} \in X^{*}$ a unique norming functional $x \in X$, that is, $\|x\|=1$ and $x^{*}(x)=\left\|x^{*}\right\|$. If $y^{\prime} \in Y^{\prime}, y^{\prime} \neq 0$, then its norming functional $y$ is in $Y$, since $y^{\prime}(P y)=P^{*} y^{*}(y)=y^{\prime}(y) \geq\|P y\|\left\|y^{\prime}\right\|$ and therefore $y=P y /\|P y\| \in Y$. Let $y_{i} \in Y$ be the norming functional of $y_{i}^{\prime}$. We show that $\left\{y_{i}\right\}$ is a block basis of $Y$. As the basis of $X$ is 1-unconditional, the support of $y_{i}$ with respect to $\left\{x_{i}\right\}$ is contained in the support of $y_{i}^{\prime}$ with respect to the basis $\left\{x_{i}^{*}\right\}$ of coordinate functionals. Therefore $\left\{y_{i}\right\}$ is a set of mutually disjoint elements of $Y$. To see that the closure of the span of the $y_{i}$ equals $Y$, let $\|x\|_{(m)}$ denote the norm of the functional $x \in X$ on $\operatorname{span}\left\{x_{i}^{*}: i>m\right\}$. As $X$ is reflexive, the basis $\left\{x_{i}^{*}\right\}$ of $X^{*}$ is shrinking, that is, $\|x\|_{(m)} \rightarrow 0$ as $m \rightarrow \infty$ for all $x \in X$ (see [19, Theorem 4.4.15]). Now let $y \in Y$. For $y^{\prime} \in Y^{\prime}$ with $\left\|y^{\prime}\right\|=1$ there are $\alpha_{i}$ such that $y^{\prime}=\sum_{i} \alpha_{i} y_{i}^{\prime}$. Since the elements in $\left\{y_{i}^{\prime}\right\}$ are disjoint relative to $\left\{x_{j}^{*}\right\}$, we have after suitable relabeling that $y_{i}^{\prime} \in \operatorname{span}\left\{x_{j}^{*}: j \geq i\right\}$. As $\left\{y_{i}^{\prime}\right\}$ is a 1 -unconditional basis, we get that

$$
\left|\left(\sum_{j} \alpha_{j} y_{j}^{\prime}\right)\left(y-\sum_{i \leq m} y_{i}^{\prime}(y) y_{i}\right)\right|=\left|\left(\sum_{i>m+1} \alpha_{i} y_{i}^{\prime}\right)(y)\right| \leq\|y\|_{(m)} \rightarrow 0
$$

as $m \rightarrow \infty$. Thus $y$ is in the closure of $\operatorname{span}\left\{y_{i}\right\}$. It follows that $\left\{y_{i}\right\}$ is a 1-unconditional basis of $Y$.

Next we show that $(T P)^{*}$ is a linear isometry from $Y^{\prime}$ onto $Y^{\prime}$. Indeed, $(T P)^{*}$ is linear and non-expansive on $X^{*}$. For $y^{\prime} \in Y^{\prime}$ and $x \in X$, $P^{*}(T P)^{*} y^{\prime}(x)=y^{\prime}\left(T P^{2} x\right)=(T P)^{*} y^{\prime}(x)$, so $(T P)^{*}$ maps $Y^{\prime}$ into $Y^{\prime}$. The same holds for $\left(T^{-1} P\right)^{*}$ and therefore $(T P)^{*}\left(T^{-1} P\right)^{*} y^{\prime}(x)=y^{\prime}\left(T^{-1} P T P x\right)=$ $P^{*} y^{\prime}(x)=y^{\prime}(x)$ for all $x \in X$, so that $(T P)^{*}$ maps $Y^{\prime}$ onto $Y^{\prime}$. To see that $(T P)^{*}$ is an isometry, let $y^{\prime} \in Y^{\prime}$ and let $y \in Y$ be its norming functional. 
Then $(T P)^{*} y^{\prime}\left(T^{-1} y\right)=y^{\prime}(y)=\left\|T^{-1} y\right\|\left\|y^{\prime}\right\|$ and $\left\|(T P)^{*} y^{\prime}\right\| \geq\left\|y^{\prime}\right\|$. Hence $(T P)^{*}$ is an isometry on $Y^{\prime}$. Proposition 3.5 and Lemma 3.6 yield that $(T P)^{*}$ is a signed permutation operator on $Y^{\prime}$ relative to the block basis $\left\{y_{i}^{\prime}\right\}$. It follows that $T$ is a signed permutation operator with respect to $\left\{y_{i}\right\}$.

To prove the last assertion we remark that if $\operatorname{dim} \operatorname{span}\left(\Omega_{f}\right)=n<\infty$, then for each $x \in X$ and $z \in \omega(x)$ we have that

$$
|\omega(x ; f)|=|\omega(z ; T)| \in \mathcal{S}(n),
$$

as $T$ is a signed permutation operator that coincides with $f$ on $\Omega_{f}$ and $\omega(x ; f)=\omega(z ; f)$ by $[8$, Theorem 1$]$.

We remark that if $X$ is finite dimensional, then $C^{2}$ smoothness of $X$ implies uniform smoothness of $X$. Moreover, the assumption that the relative interior of $\Omega_{f}$ is non-empty is always fulfilled, as $\Omega_{f}$ is a convex set in $\mathbb{R}^{n}$ in that case. Basic examples that satisfy the assumptions in Theorem 3.7 include $\ell_{p}$ spaces with $1<p<\infty$ and $p \neq 2$, vector valued $\ell_{p}\left(\ell_{q}\right)$ spaces with $p, q>2$ or $1<p, q<2$, and $\ell_{p}+\ell_{q}$ spaces with $p, q>2$.

\section{References}

[1] M. Akcoglu and U. Krengel, Nonlinear models of diffusion on a finite space. Probab. Theory Related Fields 76(4), (1987), 411-420.

[2] T. Andô, Contractive projections in $L_{p}$ spaces. Pacific J. Math. 17, (1966), 391-405.

[3] J.B. Baillon, R.E. Bruck, and S. Reich, On the asymptotic behavior of nonexpansive mappings and semigroups in Banach spaces. Houston J. Math. 4(1), (1978), 1-9.

[4] S. Banach, Théorie des opérations linéaires, Monograf. Mat., Warsaw, 1932.

[5] S.J. Bernau and H.E. Lacey, The range of a contractive projection on an $L_{p}$-space. Pacific J. Math. 53, (1974), 21-41.

[6] R.E. Bruck, Asymptotic behavior of nonexpansive mappings. Contemp. Math. 18, (1983), 1-47.

[7] R.E. Bruck, Nonexpansive projections on subsets of Banach spaces. Pac. J. Math. 47, (1973), 341-355.

[8] C.M. Dafermos and M. Slemrod, Asymptotic behavior of nonlinear contraction semigroups. J. Funct. Anal. 13, (1973), 97-106.

[9] R.G. Douglas, Contractive projections on an $L_{1}$ space. Pacific J. Math. 15, (1965), 443-462.

[10] N. Dunford and J.T. Schwartz, Linear Operators, Part 1: General Theory, Wiley Interscience, New York, 1957.

[11] M. Edelstein, On non-expansive mappings of Banach spaces. Proc. Camb. Philos. Soc. 60, (1964), 439-447. 
[12] A. L. Koldobsky, Isometries of $L_{p}\left(X ; L_{q}\right)$ and equimeasurability. Indiana Univ. Math. J. 40(2), (1991), 677-705.

[13] B. Lemmens and M. Scheutzow, A characterization of the periods of periodic points of 1-norm nonexpansive maps. Selecta Math. (N.S.) 9(4), (2003), 557-578.

[14] B. Lemmens and M. Scheutzow, On the dynamics of sup-norm nonexpansive maps. Ergodic Theory Dynam. Syst. 25(3), (2005), 861-871.

[15] B. Lemmens, M. Scheutzow, and C. Sparrow, Transitive actions of finite abelian groups of sup-norm isometries. European J. Combin. 28(4), (2007), 1163-1179.

[16] B. Lemmens, B. Randrianantoanina, and O. van Gaans, Second derivatives of norms and contractive complementation in vector-valued spaces. Studia Math. 179(2), (2007), 149-166.

[17] R. Lyons and R.D. Nussbaum, On transitive and commutative finite groups of isometries. In Fixed Point Theory and Applications (K-K. Tan ed.), pp. 189-228, World Scientific: Singapore, 1992.

[18] Yu.I. Lyubich, On the boundary spectrum of contractions in Minkowski spaces. Sib. Math. J. 11, (1970), 271-279.

[19] R.E. Megginson, An Introduction to Banach Space Theory, GTM 183, SpringerVerlag, New York, 1998.

[20] S. Mazur and S. Ulam, Sur les transformations isométriques d'espaces vectoriels normés, C. R. Acad. Sci. Paris Ser. I Math. 194, (1932), 946-948.

[21] R.D. Nussbaum, Omega limit sets of nonexpansive maps: finiteness and cardinality estimates. Differential Integral Equations 3(3), (1990), 523-540.

[22] R.D. Nussbaum, Estimates of the periodic points for nonexpansive operators. Israel J. Math. 76(3), (1991), 345-380.

[23] R.D. Nussbaum, M. Scheutzow, and S.M. Verduyn Lunel, Periodic points of nonexpansive maps and nonlinear generalizations of the Perron-Frobenius theory. Selecta Math. (N.S.) 4(1), (1998), 1-41.

[24] A.T. Plant and S. Reich, The asymptotics of nonexpansive iterations. J. Funct. Anal. 54, (1983), 308-319.

[25] R. Sine, A nonlinear Perron-Frobenius theorem. Proc. Am. Math. Soc. 109(2), (1990), 331-336.

[26] L. Tzafriri, Remarks on contractive projections in $L_{p}$-spaces. Israel J. Math. $\mathbf{7}$, (1969), 9-15.

[27] D. Weller, Hilbert's metric, part metric and self mappings of a cone, $\mathrm{PhD}$ thesis, Universität Bremen, Germany, 1987. 